\title{
Information Sources Consulted by Women in African Countries to Manage Menopausal Symptoms: A Systematic Review and Meta-Analysis
}

\author{
Kathe Hofnie-Hoëbes ${ }^{1 *}$, Alison Weightman ${ }^{2}$, Menete Shatona ${ }^{1}$, Mark Kelson ${ }^{3}$ \\ ${ }^{1}$ University of Namibia, Windhoek, Namibia \\ ${ }^{2}$ Cardiff University, Cardiff, UK \\ ${ }^{3}$ Exeter University, Exeter, UK \\ Email: ^khofnie@unam.na, WeightmanAL@cardiff.ac.uk, mshatona@unam.na, M.J.Kelson@exeter.ac.uk
}

How to cite this paper: Hofnie-Hoëbes, K., Weightman, A., Shatona, M. and Kelson, M. (2018) Information Sources Consulted by Women in African Countries to Manage Menopausal Symptoms: A Systematic Review and Meta-Analysis. Open Journal of Nursing, 8, 317-338.

https://doi.org/10.4236/ojn.2018.85027

Received: March 26, 2018

Accepted: May 28, 2018

Published: May 31, 2018

Copyright $\odot 2018$ by author and Scientific Research Publishing Inc. This work is licensed under the Creative Commons Attribution International License (CC BY 4.0).

http://creativecommons.org/licenses/by/4.0/

\begin{abstract}
Background: Despite an abundance of information regarding menopausal women in western society, African menopausal women have been overlooked in menopausal research, particularly, where they find information regarding menopause. Since inadequate health-related knowledge on menopause negatively affects the understanding of and coping with menopause and related symptoms, it is important to determine where women in Africa obtain their information about menopause. Aim: To conduct a systematic review to identify and analyse information sources consulted by menopausal women in the African region regarding the management of menopausal symptoms. Method: PubMed, Embase (OVID), Google Scholar, Medline (OVID), Medline in Process (OVID), Scopus, https://www.africabib.org/, African Index Medicus; http://indexmedicus.afro.who.int/, and African Journals Online (AJOL) were searched for publications from January 2000 to April 2017. Fourteen studies met the eligibility criteria. Twelve were quantitative while two were qualitative. Studies were independently appraised by two researchers using checklists from the Centre for Evidence-Based Management. Results: Meta-analysis explored the prevalence of different information sources reported with friends being the highest $(44 \%, 95 \%$ CI: $[0.35,0.54])$ followed by relatives $(35 \%, 95 \%$ CI: $[0.22,0.50])$ with medical providers being sources of information for only $30 \%$ of participants (95\% CI: $[0.15,0.50]$ ). Conclusion: Information regarding menopause among African women emanated primarily from friends and relatives, suggesting that women perceive menopause as a natural process whereby information is the best gleaned from informal sources. However, health care should aim to increase public awareness of menopause education and strengthen efforts to provide accurate, timely and
\end{abstract}


helpful information for women and their friends and families.

\section{Keywords}

African Countries, Identify and Analyse, Information Sources, Menopausal Symptoms, Systematic Review

\section{Introduction}

\section{1) What is already known about the topic?}

a) Sources of menopausal information are underexplored in the African region.

b) Information sources are consulted by menopausal women in the African region, but there is little information on the preferred sources of such information for the management of menopausal symptoms.

\section{2) What this paper adds}

a) Our systematic review and meta-analysis identified only fourteen eligible papers from the five African countries that were involved in the research on information sources consulted by menopausal women in the African region during 2000-2017.

b) Meta-analysis revealed six preferred information sources, with friends as the most frequently reported source of menopause information, followed by relatives; medical providers; television and radio; books, magazines, journals; and religious sources being the lowest.

\subsection{Background}

Despite an abundance of information regarding menopausal women in western society, African women have been almost overlooked in menopausal research [1] [2]. In most African states, research on menopause and how its symptoms are understood and dealt with in public policy and public health education is minimal [2] [3]. Several studies conducted in the African region confirm that awareness of information about menopause in African women was low [4] [5] [6] [7] [8].

Additionally, issues of menopause such as sources of information are under explored [9]. However, inexperience and inadequate health-related knowledge on menopause negatively affects understanding and coping with menopause and related symptoms. Equally, African menopausal women need support systems from all relevant stakeholders, including health care providers, family and society to effectively manage menopause [4] [6]. Hence, it is important to determine where women in Africa obtain information about menopause [10].

\subsection{The Purpose of the Review}

This systematic review aimed at identifying and analysing information sources consulted by menopausal women in the African region regarding the manage- 
ment of menopausal symptoms. The goal was to enable medical/health service providers to provide their clients with correct information from reliable sources and empower menopausal women to alleviate suffering during menopause. Furthermore, evidence from this review will allow policy makers, such as the ministry of health to devise appropriate interventions that will enable all women, particularly menopausal women, to receive correct information.

The specific objectives of this systematic literature review were to: review relevant African studies since 2000 to date, regarding the sources of information in the management of menopausal symptoms among pre-, peri-, menopause-, and post-menopausal women aged 35 - 90; assess the validity of the findings of the included studies and systematically analyse the relevance of information sources across the African region.

\section{Methodology}

\subsection{Definitions}

1) Menopause

Menopause is characterised by absence of menstrual period for 12 consecutive months without biological or physiological cause [4] [11] [12]. Such absence of menstrual period signifies the end of fertility and childbearing years [5] [11]. The end of fertility in a woman's life is characterised by several symptoms brought about by decreased hormonal activity [4]. The most common symptoms associated with menopause are hot flashes, decreased libido, vaginal dryness, insomnia, and bone or joint disorders [3] [5] [13] [14] [15] [16].

\section{2) Pre-menopause:}

Pre-menopause is the stage that leads up to peri-menopause. This stage affects most women in their late thirties and early forties. During this period, women are still menstruating regularly, but the estrogen and progesterone levels may begin to change [17].

\section{3) Peri-menopause}

Peri-menopause is the stage that occurs before menopause. At this stage, women have a significant drop in estrogen levels, which signifies the end of fertility in a woman's life, coupled by some troublesome symptoms, such as hot flashes, vaginal dryness and slowed metabolism. During this stage, the menstrual period becomes irregular, and women may experience missed periods, shorter menstrual cycles or suffer from longer cycles [17] [18].

\section{4) Post-menopause}

Post-menopause refers to the time after a woman's menstrual periods have ceased for 12 consecutive months. During the postmenopausal stage, many troublesome symptoms a woman may have experienced during peri-menopause gradually decrease [19].

\subsection{Methods}

\subsubsection{Review Question}

A systematic review of the literature was conducted to answer the following 
question:

What information sources are consulted by pre-, peri-, menopause-, and post-menopausal women between ages 35 - 90 in the African region in the management of menopausal symptoms since 2000 according to literature obtained from Embase (OVID), Google Scholar, MEDLINE (OVID), MEDLINE in Process (OVID), Pub Med, Scopus plus African specific databases:

https://www.africabib.org/, African Index Medicus;

http://indexmedicus.afro.who.int/, and African Journals Online (AJOL)

https://www.ajol.info/. This review was registered on 12 May 2017 in PROSPERO; the international prospective register of systematic reviews as: PROSPERO 2017 CRD42017065866 and is available from http://www.crd.york.ac.uk/PROSPERO/display_record.phd?ID=CRD420170658 $\underline{66}$

\subsubsection{Design}

A systematic literature review was conducted according to the PRISMA statement and guidelines [20].

1) Search strategy

Six databases were searched in April 2017: PubMed, BioMed Central, Embase, Google Scholar, Medline and Medline in Process and Scopus.

\section{2) Search terms}

The search strategy was designed in PubMed. In brief this comprised a combination of three sets of search terms:

Menopaus ${ }^{\star}$ [in title or abstract] OR Menopause as a Medical Subject Heading AND

(Information OR experience* OR perception* OR health seeking behav ${ }^{\star}$ OR knowledge) [In title or abstract] OR Information Seeking Behavior as a Medical Subject Heading

AND

A search filter of title/abstract terms to identify research carried out in Africa OR Africa as a Medical Subject Heading

Following testing for its ability to identify known relevant studies, the search was then adapted and run in the following additional databases: Embase (OVID), Google Scholar, Medline (OVID), Medline in Process (OVID), Scopus, www.Africabib.org, African Index Medicus; http://indexmedicus.afro.who.int/, and African Journals Online (AJOL) https://www.ajol.info/ in April 2017. The databases were chosen to provide good coverage of the African healthcare literature by including both the major global and Africa-specific resources. Complete search strategies, including combinations of text words (from title/abstract) and indexer-provided subject headings (where available), are listed in Appendix 1.

Supplementary searching was also employed: reference lists of included studies were checked for additional studies; citation tracking was used to identify more recent publications; authors of included publications were contacted to ask 
about unpublished and in-press studies.

\subsection{Inclusion and Exclusion Criteria}

To assure modern-day relevance, this study was limited to literature published from January 2000 to April 2017, when searches were completed. As this review aimed at identifying and analysing the information sources consulted by menopausal women in the Africa region, only studies that incorporated women from the African region were included. Pre-, peri-, menopause-, and post-menopausal women between ages 35 - 90 were included in this review. Complete inclusion and exclusion criteria are described in Table 1.

\subsection{Data Selection}

The results of all database searches were imported into Reference Manager 12 http://www.adeptscience.co.uk/products/refman/reference and duplicates removed. Records potentially meeting the inclusion criteria were screened by two researchers, independently, first at title/abstract stage then at full text stage. Additional studies obtained from cross referencing and reviews met the inclusion criteria and were included in the full text review. Data were then independently coded by two researchers based on the sources of information used by the menopausal women.

\subsubsection{Quality Assessment}

Two researchers firstly independently reviewed different appraisal tools and then agreed on the Center for Evidence-Based Management (CEBMa) critical appraisal tools, designed for qualitative and cross-sectional studies (https://www.cebma.org/resources-and-tools/what-is-critical-appraisal). The CEBMa critical appraisal tools have 10 (qualitative) and 12 (quantitative) yes/no questions depending on the type of study to help guide the assessment of the quality of the literature being critiqued. Quantitative studies were critiqued,

Table 1. Inclusion and exclusion criteria for the review.

Menopausal women in Africa. These were all women who were experiencing amenorrhoea for 12 consecutive months
and above without biological or physiological cause.
Pre-menopause: Refers to the stage that leads up to peri-menopause. This stage affects most women in their late
thirties and early forties.
Peri-menopause: Peri-menopause is the stage that occurs before menopause. During this stage, the menstrual period
becomes irregular, which signifies the end of fertility in woman's life, coupled by some troublesome symptoms.
Post-menopause: Refers to the time after a woman's menstrual periods have ceased for 12 consecutive months. During
postmenopausal stage, many troublesome symptoms a woman may have experienced during peri- menopause gradually
decrease.
Use of information sources to understand and manage signs and symptoms. [Information refers to knowledge
communicated or received concerning a particular fact or circumstances]
1) Hot flashes
2) Sexual function
3) All other menopause related signs and symptoms
1) Include publications since 2000 published in the African region
2) Exclude papers that discuss a lack of knowledge/information in general but with no description of information
sources used.


based on the following criteria: focused question, study design, selection of participants, selection bias, representativeness, sample size by power calculation, response rate, question reliability, statistical significance, confidence intervals, confounders, relevance to African population, while qualitative studies were critiqued on: focused question, study design, study context, fieldwork, evidence inspected, procedure reliability, analysis reliability, results credibility, conclusions justified, findings transferable. (See comments in Table 2) Thereafter, eligible studies (14) were independently appraised by two researchers. The researchers had one Skype call and one in-person meeting and discussed appraised articles. Two disagreements in the scores were discussed until consensus was reached.

\subsubsection{Data Extraction}

Tables were used to extract author name, year of publication, country of origin, aim of study, study design, sample characteristics, data collection instruments and data collection procedure, results and limitations identified via the quality assessment process, as shown in Table 3.

\subsection{Analysis}

Prevalence information was pooled with random effects meta-analysis where appropriate. Results are presented with the associated fixed effect estimates and 95\% confidence interval as well as the i-square statistic. All analysis was conducted using the $\mathrm{R}$ programming language and environment implementing the meta and metafor packages [21] [22] [23].

\section{Results}

Our initial search yielded 794 studies (Figure 1). Twelve additional studies were

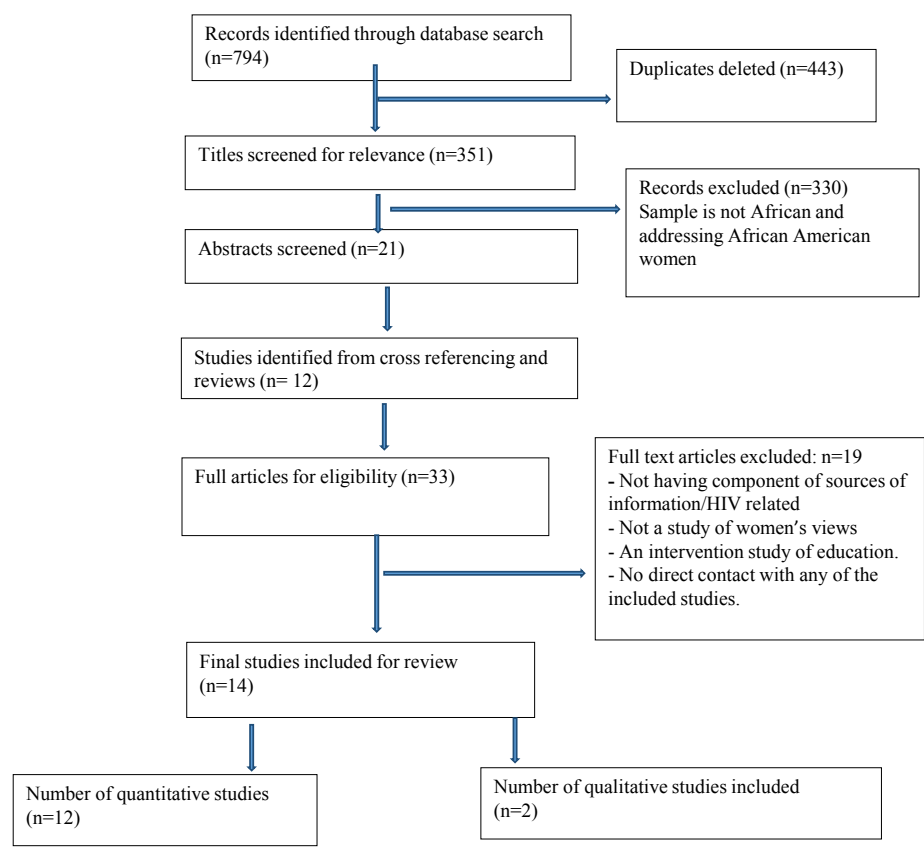

Figure 1. Flow diagram of reviewed studies. 
identified from cross referencing and reviews. After 443 duplicates were deleted from the initial search, the remaining 351 studies together with the twelve records through other sources were then imported into Reference Manager 12. Three hundred and thirty (330) studies were excluded because they were conducted primarily among African American menopausal women in America. The 21 full text review records plus the twelve additional records were retained. Thirty three full-text articles were assessed for eligibility (Appendix 2), and 19 records excluded, with reasons including: not providing sources of information, HIV related, not a study of women's views, or an educational interventional study. The eligibility criteria were then reapplied to the remaining 14 full texts for thematic synthesis to illustrate the key information sources, used by menopausal women in the African region. Of the 14 reviewed studies, 12 employed quantitative and 2 qualitative approaches. Resultant studies are summarised in Table 2.

\subsection{Reporting}

The majority of the studies (12) employed a quantitative cross-sectional design: two (2) studies were qualitative in nature. The information from the cross-sectional studies was collected with structured questionnaires, using close and open ended questions, while interviews were used for qualitative studies. Response rates were between $75-100 \%$. The fourteen eligible studies reporting on the information sources used by menopausal women in the African region were from Nigeria (7), South Africa (3), Ethiopia (2), Namibia (1) and Ghana (1). Meta-analysis was conducted to pool the prevalence of six different information sources: friends, relatives, medical providers, books/magazines/journals, TV/radio and religious sources.

Friends were the most frequently reported source of menopause information with a point prevalence estimate of 0.44 (95\% CI: [0.35, 0.54], number of studies = 7), followed by relatives with a point prevalence estimate of 0.35 (95\% CI: $[0.22,0.50]$, number of studies $=5$ ). Medical providers were next, prevalence 0.30 (95\% CI: $[0.15,0.50]$, number of studies $=9$ ), followed by TV and radio, prevalence 0.20 ( $95 \%$ CI: $[0.06,0.48]$ number of studies $=5$ ), then books, magazines, journals prevalence 0.19 (95\% CI: $[0.10,0.33$, number of studies $=9$ ), with religious sources being the lowest, prevalence 0.06 (95\% CI: [0.02, 0.14], number of studies $=5$ ). It must be noted however that all of the above analyses exhibited high levels of heterogeneity ( $\mathrm{i}^{2}$ all above $\left.94 \%\right)$.

\subsection{Quality Assessment}

Fourteen studies were appraised. Each of the critiqued studies had $10-12$ components needed to constitute a strong study, out of 10 - 12 possible criteria (see Table 2). According to the results of the critical appraisals, studies were overall of good quality. Survey instruments were rarely piloted or validated however, and studies did not generally assess statistical significance intervals because these studies were mostly addressing perceptions, attitudes and or behaviour of the 
Table 2. Summary of quality assessment based on CEBMa tools.

(a)

\begin{tabular}{|c|c|}
\hline $\begin{array}{l}\text { Summary of appraised quantitative papers } \\
\qquad(\mathrm{n}=12)\end{array}$ & $\begin{array}{l}\text { Number of studies meeting this criteria } \\
\qquad \mathrm{n}=12 \text { total studies (\%) }\end{array}$ \\
\hline \multicolumn{2}{|l|}{ Clearly described: } \\
\hline Focused question: & $12(100)$ \\
\hline Study design & $12(100)$ \\
\hline Selection described: & $10(83 \%)$ \\
\hline $\begin{array}{l}\text { Low risk of selection bias } \\
\text { Low risk: } 8 \text { papers }\end{array}$ & $8(67 \%)$ \\
\hline $\begin{array}{l}\text { Representative of group } \\
\text { indicated: } 6 \text { papers }\end{array}$ & $6(50 \%)$ \\
\hline $\begin{array}{l}\text { Appropriate sample size by Power } \\
\text { Statistical Power indicated: } 2 \text { papers }\end{array}$ & $2(17 \%)$ \\
\hline $\begin{array}{c}\text { Response rate } \geq 70 \% \\
\text { More than } 70 \%: 6 \text { papers }\end{array}$ & $6(50 \%)$ \\
\hline $\begin{array}{l}\text { Reliable measurement } \\
\text { Reliable:8 papers }\end{array}$ & $8(67 \%)$ \\
\hline $\begin{array}{l}\text { Statistical significance } \\
\text { Not assessed in all (12) papers }\end{array}$ & 0 \\
\hline $\begin{array}{l}\text { Confidence intervals } \\
\text { Not provided: in all } 12 \text { papers }\end{array}$ & 0 \\
\hline $\begin{array}{l}\text { No other confounders } \\
\text { Confounders not obvious } \\
\text { in all } 12 \text { papers }\end{array}$ & $12(100 \%)$ \\
\hline $\begin{array}{l}\text { Relevance to African population } \\
\text { Relevant: } 9 \text { papers }\end{array}$ & $9(75 \%)$ \\
\hline
\end{tabular}

(b)

\begin{tabular}{cc}
$\begin{array}{c}\text { Summary of appraised qualitative papers } \\
(\mathbf{n}=\mathbf{2})\end{array}$ & $\begin{array}{c}\text { Number of studies meeting this criteria } \\
\mathbf{n}=10 \text { total studies }(\%)\end{array}$ \\
\hline Clearly described: & $2(100 \%)$ \\
\hline Focused question: & $2(100 \%)$ \\
Study design & $2(100 \%)$ \\
Study context & $1(50 \%)$ \\
Fieldwork & $1(50 \%)$ \\
Evidence inspected & $2(100 \%)$ \\
Procedure reliability & 0 \\
Analysis reliability & $2(100 \%)$ \\
Results credibility & $2(100 \%)$ \\
Conclusions justified & 0 \\
Findings transferable. & 0 \\
\hline
\end{tabular}

African menopausal women.

\section{Discussion}

This review synthesised the data regarding information sources consulted by 
Table 3. Included studies and data extraction.

\begin{tabular}{|c|c|c|c|c|}
\hline Study details & Population \& setting & Methods & $\begin{array}{l}\text { Results: Information } \\
\text { sources consulted }\end{array}$ & Limitations \\
\hline $\begin{array}{l}\text { First author and year: } \\
\text { Anolue, } 2012 \\
\text { Study design: } \\
\text { Quantitative - } \\
\text { Questionnaire } \\
\text { Objective: } \\
\text { To document women's } \\
\text { experience of menopause } \\
\text { in rural communities and } \\
\text { compare this with urban } \\
\text { centres }\end{array}$ & $\begin{array}{l}\text { Setting: } \\
\text { Nigeria } \\
\text { Participants: } \\
\mathrm{N}=349 \text {. Mean age } 58 \text { years } \\
\text { How recruited: } \\
\text { Random selection from } 13 \text { autonomous } \\
\text { rural communities } \\
\text { When: } \\
\text { August } 2009 \\
\text { Inclusion criteria: } \\
\text { Menopausal women with at least } 12 \\
\text { months amenorrhoea. All participants } \\
\text { from a certain ethnic group }\end{array}$ & $\begin{array}{l}\text { Tools and data } \\
\text { collection: } \\
\text { Questionnaire } \\
\text { Analysis: } \\
\text { SPSS software. } \\
\text { Chi-square test }\end{array}$ & $\begin{array}{l}\text { Peers }=51.3 \% \\
\text { Parents }=28.9 \% \\
\text { Others }=12.0 \% \\
\text { Church group }=7.7 \%\end{array}$ & $\begin{array}{l}\text { Total population from } \\
\text { where the sample was } \\
\text { drawn not indicated } \\
\text { Tools not validated } \\
\text { Design not indicated }\end{array}$ \\
\hline $\begin{array}{l}\text { First author and year: } \\
\text { Bello, } 2016 \\
\text { Study design: } \\
\text { Quantitative - } \\
\text { Questionnaire } \\
\text { Objective: } \\
\text { To determine level of } \\
\text { awareness and perceptions } \\
\text { about menopause and sex } \\
\text { in peri-menopausal } \\
\text { women }\end{array}$ & $\begin{array}{l}\text { Setting: } \\
\text { Nigeria, Family Medicine Department } \\
\text { in Ibadan hospital } \\
\text { Participants: } \\
\mathrm{N}=352 \text {. Aged } \geq 40 \text { years (mean } 46.3 \text { ). } \\
\text { How recruited: } \\
\text { Not described } \\
\text { When: } \\
\text { Not stated } \\
\text { Inclusion criteria: } \\
\text { Peri-menopausal women. Menopausal } \\
\text { and post-menopausal women (12 } \\
\text { months or more amenorrhoea) } \\
\text { excluded }\end{array}$ & $\begin{array}{l}\text { Tools and data } \\
\text { collection: } \\
\text { Structured } \\
\text { questionnaire } \\
\text { Analysis: } \\
\text { SPSS software. } \\
\text { Chi-square test }\end{array}$ & $\begin{array}{l}\text { Friends \& Peers }= \\
45.7 \% \\
\text { Health care providers }= \\
17.0 \% \\
\text { Aunts \& older sisters = } \\
15.1 \% \\
\text { Doctors }=13.6 \% \\
\text { TV } / \text { Radio }=12.2 \% \\
\text { Books \& other print } \\
\text { media }=12.2 \% \\
\text { Others }=1.4 \%\end{array}$ & $\begin{array}{l}\text { Data collection tools } \\
\text { not subjected to validity } \\
\text { and reliability test } \\
\text { Data collection period } \\
\text { not indicated } \\
\text { Selection method not } \\
\text { indicated } \\
\text { Total population not } \\
\text { described }\end{array}$ \\
\hline $\begin{array}{l}\text { First author and year: } \\
\text { Dienye, } 2013 \\
\text { Study design: } \\
\text { Quantitative - } \\
\text { Questionnaire } \\
\text { Objective: } \\
\text { To determine frequency \& } \\
\text { severity of menopausal } \\
\text { symptoms and health } \\
\text { seeking behavior of } \\
\text { women with menopausal } \\
\text { symptoms }\end{array}$ & $\begin{array}{l}\text { Setting: } \\
\text { Nigeria, Port Harcourt Teaching } \\
\text { Hospital } \\
\text { Participants: } \\
\mathrm{N}=385 \text {. Aged } 35-95 \text { years (mean } 58.4 \text { ) } \\
\text { How recruited: } \\
\text { Consecutive recruitment at clinic until } \\
\text { pre-specified sample size reached } \\
\text { When: } \\
\text { July-September } 2010 \\
\text { Inclusion criteria: } \\
\text { Postmenopausal women with at least } 12 \\
\text { months amenorrhoea }\end{array}$ & $\begin{array}{l}\text { Tools and data } \\
\text { collection: } \\
\text { Questionnaire results } \\
\text { recorded in Microsoft } \\
\text { Office Excel } \\
\text { Analysis: } \\
\text { Epi Info software }\end{array}$ & $\begin{array}{l}\text { Patent drug } \\
\text { dealers }=51.4 \% \\
\text { Health workers }=44.7 \% \\
\text { Nothing }=26.5 \% \\
\text { Religious leaders }= \\
20.3 \% \\
\text { Traditional } \\
\text { healers }=3.8 \%\end{array}$ & $\begin{array}{l}\text { Validity of tools not } \\
\text { measured } \\
\text { Missing data was not } \\
\text { accounted for }\end{array}$ \\
\hline $\begin{array}{l}\text { First author and year: } \\
\text { Eshetu, } 2015 \\
\text { Study design: } \\
\text { Quantitative - } \\
\text { Questionnaire } \\
\text { Objective: } \\
\text { To assess level of } \\
\text { knowledge and attitude of } \\
\text { women aged } 30-49 \mathrm{yrs}\end{array}$ & $\begin{array}{l}\text { Setting: } \\
\text { Ethiopia, Addis Ababa } \\
\text { Participants: } \\
\mathrm{N}=599 \text { ( } 586 \text { correctly completed). } \\
\text { Aged } 30 \text { - } 49 \text { years (mean } 37 \text { ) } \\
\text { How recruited: } \\
\text { Multi-stage random sampling from } \\
\text { community until pre-specified sample } \\
\text { size reached } \\
\text { When: } \\
\text { April-May } 2015 \\
\text { Inclusion criteria: } \\
\text { Age, permanent residency, consent }\end{array}$ & $\begin{array}{l}\text { Tools and data } \\
\text { collection: } \\
\text { Questionnaire } \\
\text { Analysis: } \\
\text { SPSS software. Logis- } \\
\text { tic regression. }\end{array}$ & $\begin{array}{l}\text { Friend }=37.7 \% \\
\text { Relatives }=31.7 \% \\
\text { Medical care } \\
\text { providers }=20.8 \% \\
\text { Books, Magazines, } \\
\text { Journals }=14.8 \% \\
\text { Mass media (Radio, } \\
\text { TV) }=14.7 \% \\
\text { Educational sessions = } \\
7.0 \% \\
\text { Community Elder }= \\
1.4 \%\end{array}$ & $\begin{array}{l}\text { Validity of tools not } \\
\text { indicated }\end{array}$ \\
\hline
\end{tabular}




\section{Continued}

First author and year:

Hofnie-//Hoëbes, 2009

Study design:

Mixed methods -

Quantitative

(questionnaire) and

qualitative (open ended

interviews)

Objective:

To determine where and

available regarding

menopause and from

where it is obtained

First author and year:

Ibraheem, 2015

Study design:

Mixed methods -

Quantitative

(questionnaire) and

qualitative (focus groups)

Objective:

To determine menopausal

experiences and

perceptions of women what information is

\section{Setting:}

Namibia, urban and rural locations

Participants:

$\mathrm{N}=1079$

How recruited:

Multi-stage cluster sampling from

community

When:

March-April 2008

Inclusion criteria:

Pre-menopausal, peri-menopausal and

menopausal women

\section{Setting:}

Nigeria

Participants:

$\mathrm{N}=240$. Aged $46-69$ years (mean

56.9)

How recruited:

Multi-stage random sampling from list

of local government areas

When:

April 2008

Inclusion criteria:

Age, amenorrhoea for 12 months \&

above, member of community \&

voluntary verbal consent

Setting:

Nigeria

Participants:

$\mathrm{N}=432$. Aged $45-60$

How recruited:

Random selection

When:

Not stated

Inclusion criteria:

Not described

\section{Setting:}

South Africa, Durban

Participants:

$\mathrm{N}=150$ (complete data and

included = 139). Aged $18-40$ years

(mean 31)

How recruited:

Not stated

When:

Not stated menopause/HRT to

Inclusion criteria:

engage in patient-provider discussions and utilised in full informed consent for

40 years.
Tools and data

collection:

Questionnaire and

open-ended

interviews with a

guide

Analysis:

SPSS software.

Pearson chi-square test. Descriptive do-

cumentation of focus

group discussion

Tools and data

collection:

Piloted guide for

focus group

discussions with

results informing

semi-structured

questionnaires.

Cronbach's Alpha for

instrument 0.745

Analysis:

Chi-square and

descriptive for

quantitative data.

Content analysis for

focus groups.

Tools and data

collection:

Self-administered

structured

questionnaire

Analysis:

SPSS software.

Descriptive statistics

with cross

tabulations.

No comment $=44.4 \%$

Gynaecology

clinic $=22.2 \%$

Self prayers $=13.9 \%$

Medical clinic in

hospital $=11.1 \%$

Native doctor $=2.8 \%$

Clinic and priest and

self prayer $=2.8 \%$

priest $=2.8 \%$
Validity and reliability

of tools not clear
Medical clinic and

No exclusion criteria

Menopausal status not indicated

Data collection tools not subjected to validity and reliability test Data collection period not indicated

Total population not described

Cross-tabs indicated in methods, but not seen in data analysis

Sample not proportional

Tools and data

Family $=52.5 \%$

Friends $=44.6 \%$

collection:

Questionnaire

Analysis:

ANOVA chi-square

Media $=41.7 \%$

GPs $=14.4 \%$

Clinic: $11.5 \%$

Hospital $=9.4 \%$
Design not indicated

Aim too broad

Tools not subjected to

reliability test

Initial inclusion of

teenagers was

inadequate 


\begin{tabular}{|c|c|c|c|c|}
\hline $\begin{array}{l}\text { First author and year: } \\
\text { Odiari, } 2012 \\
\text { Study design: } \\
\text { Qualitative - Interviews } \\
\text { Objective: } \\
\text { To investigate how women } \\
\text { in low resource setting } \\
\text { manage menopausal } \\
\text { symptoms without HRT }\end{array}$ & $\begin{array}{l}\text { Setting: } \\
\text { Ghana, Accra regional hospital } \\
\text { Participants: } \\
\mathrm{N}=34 \text { (meeting criteria from } 82 \\
\text { considered). Mean age } 58 \text { years } \\
\text { How recruited: } \\
\text { Purposive sampling from clinic with } \\
\text { random approach for inclusion } \\
\text { When: } \\
\text { Not stated } \\
\text { Inclusion criteria: } \\
\text { Had experienced natural menopause. } \\
\text { No history of cardiac disease or } \\
\text { arthritis before menopause. Conversant } \\
\text { in English or Ghanaian languages }\end{array}$ & $\begin{array}{l}\text { Tools and data } \\
\text { collection: } \\
\text { Semi-structured } \\
\text { interview guide. Data } \\
\text { collection suspended } \\
\text { when data saturation } \\
\text { was reached } \\
\text { Analysis: } \\
\text { NVivo } 8 \text { software. } \\
\text { Chi-square test. } \\
\text { Content analysis. }\end{array}$ & $\begin{array}{l}\text { People who previously } \\
\text { experienced menopause } \\
\text { (Friends, colleagues or } \\
\text { family) = 55.9\% } \\
\text { Health care } \\
\text { providers/media/books/ } \\
\text { church meetings = } \\
44.1 \%\end{array}$ & $\begin{array}{l}\text { No exclusion criteria } \\
\text { Random sampling } \\
\text { process unclear } \\
\text { Not clear whether two } \\
\text { researchers were } \\
\text { involved in data } \\
\text { analysis }\end{array}$ \\
\hline $\begin{array}{l}\text { First author and year: } \\
\text { Osinovo, } 2003 \\
\text { Study design: } \\
\text { Quantitative - } \\
\text { Questionnaire } \\
\text { Objective: } \\
\text { To investigate factors } \\
\text { associated with attitude } \\
\text { towards sex-role, } \\
\text { self-image \& marital } \\
\text { satisfaction on } \\
\text { psychological health } \\
\text { status, perception of } \\
\text { menopause and sexual } \\
\text { satisfaction }\end{array}$ & $\begin{array}{l}\text { Setting: } \\
\text { Nigeria, Urban } \\
\text { Participants: } \\
\mathrm{N}=45 \text {. Aged } 36 \text { - } 70 \text { years (mean } 51.4 \text { ) } \\
\text { How recruited: } \\
\text { Purposive sampling from women } \\
\text { attending a seminar on aging } \\
\text { When: } \\
\text { Not stated } \\
\text { Inclusion criteria: } \\
\text { Non-menopausal and menopausal }\end{array}$ & $\begin{array}{l}\text { Tools and data } \\
\text { collection: } \\
\text { Piloted questionnaire } \\
\text { Analysis: } \\
\text { SPSS software. } \\
\text { Coefficient alpha: } \\
\text { 0.94. Descriptive stats }\end{array}$ & $\begin{array}{l}\text { Not stated }=36 \% \\
\text { Health } \\
\text { institutions: }=22 \% \\
\text { Books }=11 \% \\
\text { Doctors }=7 \% \\
\text { Books } / \text { Health } \\
\text { workers }=7 \% \\
\text { Friends }=4 \% \\
\text { Books/friends/relatives } \\
=4 \% \\
\text { Books/Friends/Doctors } \\
=4 \% \\
\text { Professional } \\
\text { experience }=2 \% \\
\text { Sex education }=2 \%\end{array}$ & $\begin{array}{l}\text { Not clear how many } \\
\text { questionnaires were } \\
\text { distributed in total }\end{array}$ \\
\hline $\begin{array}{l}\text { First author and year: } \\
\text { Ramakeula, } 2015 \\
\text { Study design: } \\
\text { Qualitative - Focus groups } \\
\text { Objective: } \\
\text { To explore and describe } \\
\text { women, menopause and } \\
\text { aging in rural villages }\end{array}$ & $\begin{array}{l}\text { Setting: } \\
\text { South Africa, rural } \\
\text { Participants: } \\
\mathrm{N}=24 \text { to } 32 \text { (not specified). Aged } 45 \\
\text { and over. } \\
\text { How recruited: } \\
\text { Purposive sampling in four villages } \\
\text { When: } \\
\text { Not stated } \\
\text { Inclusion criteria: } \\
\text { Pre-, peri- and post-menopausal } \\
\text { women }\end{array}$ & $\begin{array}{l}\text { Tools and data } \\
\text { collection: } \\
\text { Four focus groups (6 } \\
\text { - } 8 \text { women per group) } \\
\text { Analysis: } \\
\text { Tesch's open coding. } \\
\text { Clustered into themes } \\
\text { and sub-themes. }\end{array}$ & $\begin{array}{l}\text { Informed by nurse at a } \\
\text { church conference }\end{array}$ & $\begin{array}{l}\text { Data collection period } \\
\text { not indicated } \\
\text { Not indicated if two } \\
\text { researchers were } \\
\text { involved in data } \\
\text { analysis }\end{array}$ \\
\hline $\begin{array}{l}\text { First author and year: } \\
\text { Saka, } 2011 \\
\text { Study design: } \\
\text { Quantitative - } \\
\text { Questionnaire } \\
\text { Objective: } \\
\text { To assess the perceived } \\
\text { cause, expectation, } \\
\text { knowledge and sources of } \\
\text { health information, } \\
\text { women's knowledge and } \\
\text { adjustment behaviour } \\
\text { towards menopause. }\end{array}$ & $\begin{array}{l}\text { Setting: } \\
\text { Nigeria, Ilorin } \\
\text { Participants: } \\
\mathrm{N}=400 \text { ( } 396 \text { providing information on } \\
\text { sources used). Aged } 40 \text { years and over } \\
\text { How recruited: } \\
\text { Multi-stage random sampling } \\
\text { When: } \\
\text { Not stated } \\
\text { Inclusion criteria: } \\
\text { Menopausal women with } 12 \text { months } \\
\text { amenorrhoea }\end{array}$ & $\begin{array}{l}\text { Tools and data } \\
\text { collection: } \\
\text { Semi-structured } \\
\text { questionnaire } \\
\text { translated from } \\
\text { English to other } \\
\text { languages as required } \\
\text { Analysis: } \\
\text { EpiInfo software. } \\
\text { Means and } \\
\text { frequencies. } \\
\text { Chi-square test. }\end{array}$ & $\begin{array}{l}\text { Friends }=38.6 \% \\
\text { Relatives }=28.0 \% \\
\text { Group meeting \& } \\
\text { religious home }=12.6 \% \\
\text { Health workers }=9.6 \% \\
\text { Books/news } \\
\text { papers } / \text { magazines } / \text { mass } \\
\text { media }=7.1 \% \\
\text { Others }=4.0 \%\end{array}$ & $\begin{array}{l}\text { Data collection period } \\
\text { not indicated } \\
\text { Validity and reliability } \\
\text { of tools not indicated }\end{array}$ \\
\hline
\end{tabular}




\section{Continued}

\begin{tabular}{|c|c|c|c|c|}
\hline $\begin{array}{l}\text { First author and year: } \\
\text { Smith, } 2009 \\
\text { Study design: } \\
\text { Quantitative - } \\
\text { Questionnaire } \\
\text { Objective: } \\
\text { To evaluate current patient } \\
\text { perspectives on the } \\
\text { menopausal period in } \\
\text { South Africa }\end{array}$ & $\begin{array}{l}\text { Setting: } \\
\text { South Africa, private specialist practice } \\
\text { in Tygerberg } \\
\text { Participants: } \\
\mathrm{N}=410 \text { ( } 541 \text { distributed, } 421 \text { returned, } \\
410 \text { used). Aged } 37 \text { - } 88 \text { years (mean } \\
59.9 \text { ) } \\
\text { How recruited: } \\
\text { Purposive sampling } \\
\text { When: } \\
\text { Jan-July } 2003 \\
\text { Inclusion criteria: } \\
\text { All known patients with established } \\
\text { post-menopausal status using hormone } \\
\text { therapy }\end{array}$ & $\begin{array}{l}\text { Tools and data } \\
\text { collection: } \\
\text { Postal questionnaire } \\
\text { Analysis: } \\
\text { SPSS software. Means } \\
\text { (standard deviations) }\end{array}$ & $\begin{array}{l}\text { Gynaecologists }=87.2 \% \\
\text { GPs }=28.3 \% \\
\text { Print \& electronic } \\
\text { media }=38.3 \%\end{array}$ & $\begin{array}{l}\text { Data collection tools } \\
\text { not subjected to validity } \\
\text { and reliability test } \\
\text { Single private practice } \\
\text { included but total } \\
\text { population of private } \\
\text { practices unknown }\end{array}$ \\
\hline $\begin{array}{l}\text { First author and year: } \\
\text { Tsehay, } 2014 \\
\text { Study design: } \\
\text { Quantitative - } \\
\text { Questionnaire } \\
\text { Objective: } \\
\text { To examine middle aged } \\
\text { women's experience and } \\
\text { attitudes towards } \\
\text { menopausal symptoms }\end{array}$ & $\begin{array}{l}\text { Setting: } \\
\text { Ethiopia, Danglia Town } \\
\text { Participants: } \\
\mathrm{N}=263 \text { pre- and post-menopausal } \\
\text { women. } 35 \text { - } 70 \text { years (mean } 46.7 \text { ) } \\
\text { How recruited: } \\
\text { Purposive random sampling. } \\
\text { When: } \\
\text { Not stated } \\
\text { Inclusion criteria: } \\
\text { Women's menstrual status }\end{array}$ & $\begin{array}{l}\text { Tools and data } \\
\text { collection: } \\
\text { 1) Semi -Structured } \\
\text { questionnaires } \\
\text { 2) Piloted } \\
\text { 3) Interviews } \\
\text { 4) Questionnaires } \\
\text { assessed by experts } \\
\text { for content \& } \\
\text { Cronbach's Alpha: }= \\
0.87 \text { for symptoms } \\
\text { and } 0.79 \text { for attitudes } \\
\text { Analysis: } \\
\text { SPSS software. } P \text { val- } \\
\text { ue = } 0.05\end{array}$ & $\begin{array}{l}\text { Friends }=70.8 \% \\
\text { Medical care providers } \\
=68.8 \% \\
\text { Books, magazines, } \\
\text { journals }=66.7 \% \\
\text { Relatives }=56.3 \% \\
\text { Educational sessions = } \\
43.8 \% \\
\text { Community Elders = } \\
22.9 \%\end{array}$ & $\begin{array}{l}\text { Data collection period } \\
\text { not indicated } \\
\text { Ethical considerations } \\
\text { not indicated }\end{array}$ \\
\hline
\end{tabular}

menopausal women in the African region. The available literature was of high quality, despite the heterogeneity of the methodologies and sample characteristics and assessment tools.

Based on the findings and consistent with previous reports [24], African menopausal women are more likely to learn about the menopause from their friends and relatives, rather than from professional assistance. Religious sources were the lowest of all that were consulted by women in the African region about the menopause.

The meta-analyses revealed high heterogeneity indicating that it is unlikely that the studies were all measuring the same quantities in exactly the same way. This is perhaps unsurprising given the wide geographical area covered by this study, encompassing many different cultures. The results must therefore be interpreted carefully.

Although women experience menopause in a universal manner due to decline in ovarian function leading to different menopausal symptoms, the actual experiences of the most common symptoms such as hot flushes, night sweats and low libido are individual [6] [13] [14] [25] [26]. Menopausal women may also experience challenges such as: anxiety, poor-self-image, low self-esteem, sleep- 
lessness, panic [4], due to the multidimensional nature of menopause, which is influenced by biological, psychological, social and cultural dimensions [5]. Therefore, lack of professional awareness may contribute to silent suffering of menopausal women [2] [4]. This is particularly true in the majority of African states where discussion of reproductive health issues are considered taboo, private and sensitive and therefore not easily shared amongst others [25] [27].

It is equally desirable that women have access to information on the menopause from health care providers regarding current treatment options [28] [29] [30] and associated risks and benefits of menopause. Medical professionals are best placed to provide menopausal women with individualised care to improve quality of life. Regarding some common menopausal symptoms, such as reduced sexual functioning, health care workers should advise women that pharmacological and non-pharmacological therapies are available to treat atrophic vaginitis and relieve pain during intercourse [28]. With over three quarters of women not citing medical professionals as an information source there is clearly room for improvement.

Given how many women access menopause information through friends and relatives, it may be helpful for health care providers to design special programmes to inform them about menopause; as partners or family members, men also may benefit from education as they experience menopausal symptoms alongside these women without prior knowledge or understanding. Such awareness will help family and friends to be sensitive to menopausal women during this period and provide adequate support [31]. The public health systems should also mobilise adequate resources to improve the awareness and knowledge of the women about menopause and menopausal symptoms to promote active and healthy living during the period of menopause [32]. Moreover, clinical research regarding menopause and understanding of menopausal symptoms should be done in the African region to guide public health policy and education.

\section{Limitations}

Although a comprehensive search was undertaken, we cannot guarantee that all relevant research studies were identified. All the papers that discussed lack of knowledge/information in general but with no description of information sources were excluded. These criteria might have limited the scope of available literature on the information sources in the African region.

\section{Conclusion}

Women in African countries experiencing menopause are more likely to have gained information about their condition from friends and relatives than from health professionals. Consequently, while relatives and friends might support them during this uncertain period, there is a clear opportunity for medical professionals to play a greater role in the education of women experiencing the menopause. 


\section{Acknowledgements}

Special thanks are extended to Writing to Improve Nursing Science (WINS) Program (Columbia University, School of Nursing) who provided us with fundamental information throughout the course of this paper, with special reference to Dr Carolyn Sun (Associate Research Scientist), who mentored this review. We also acknowledge the support of the Phoenix Project (a University of Namibia and Cardiff University partnership, led by Professor Judith Hall) for this international collaboration.

\section{Ethical Approval}

As this was a review of existing literature, no ethical approval was required.

\section{References}

[1] Tsehay, D.S., Mulatie, M.M. and Sellakumar, G.M. (2014) Determinants of Menopausal Symptoms and Attitudes among Middle Aged Women: The Case of Dangila Town, North West Ethiopia. Innovare Journal of Social Sciences, 2, 15-20.

[2] Ramakuela, N.J., Khoza, L.B. and Akinsola, H.A. (2012) Menopausal Challenges as Perceived by Women in Rural Villages of Limpopo Province, South Africa. African Journal for Physical, Health Education, Recreation and Dance, 240-258.

[3] Hofnie-Hoëbes, K., Matengu, K. and Iipinge, S. (2009) An Investigation and Assessment of Knowledge of Menopause, Menopausal Symptoms and Issues among Women and Men in Namibia. University of Namibia, Multidisciplinary Research Centre Social Sciences Division. (Unpublished)

[4] Dimkpa, D.I. (2011) Psychosocial Adjustment Needs of Menopausal Women. International Multidisciplinary Journal, Ethiopia, 5, 288-302. https://doi.org/10.4314/afrrev.v5i5.23

[5] Ama, N.O. and Ngome, E. (2013) Menopauseal Perceptions and Experiences of Older Women from Selected Sites in Botswana. Advances in Sexual Medicine, 3 47-59. https://doi.org/10.4236/asm.2013.33009

[6] Makuwa, G.N., Rikhotso, S.R. and Mulaudzi, F.M. (2015) The Perception of African Women Regarding Natural Menopause in Mamelodi, Tshwane District. Curationis, 38, 1531. https://doi.org/10.4102/curationis.v38i2.1531

[7] Saka, M.J., Akande, T.M., Saka, A.O., Odusolu, P.O., Latinwo, A.W.O., Jimoh, A.A.G. and Raji, H.O. (2014) Assessment of Expectation, Knowledge and Adjustment Behaviour of Menopausal Women in North Central Nigeria. The Tropical Journal of Health Sciences, 18, 59-64.

[8] Osinowo, H.O. (2003) Psychosocial Factors Associated with Perceived Psychological Health, Perception of Menopause and Sexual Satisfaction in Menopausal Women and Controls. West African Journal of Medicine, 22, 225-231.

[9] Odiari, E.A. and Chambers, A.N. (2012) Perceptions, Attitudes and Self-Management of Natural Menopausal Symptoms in Ghanaian Women. Health Care for Women International, 33, 560-574. https://doi.org/10.1080/07399332.2012.655393

[10] Maharaj, N.R., Gangaram, R. and Moodley, J. (2007) The Menopause, Hormone Replacement Therapy and Informed Consent: Are Women in an Under-Resourced Country Adequately Aware? Journal of Obstetrics and Gynecology, 27, 300-304. https://doi.org/10.1080/01443610701227943 
[11] Bello, A.B. and Daramola, O.O. (2016) Attitude to the Menopause and Sex amongst Middle-Aged Woman in a Family Medicine Clinic in Ibadan, Nigeria. Obstetrics and Gynecology International, 2016, Article ID: 203105. https://doi.org/10.1155/2016/2031056

[12] Dienye, P.O., Judah, F. and Ndukwu, G. (2013) Frequency of Symptoms and Health Seeking Behaviour of Menopausal Women in an Out-Patient Clinic in Port Harcourt, Nigeria. Global Journal of Health Science, 5, 39-47. https://doi.org/10.5539/gjhs.v5n4p39

[13] Mbu, R.E., Abauleth, Y.R., Koffi, A., Keita, N., Dolo, A. and Lankoande, J. (2014) Effect of Daily Supplementation of Soy Isoflavones on Hot Flashes and Night Sweats in African Menopausal Women. Open Journal of Obstetrics and Gynecology, 4, 42-46. https://doi.org/10.4236/ojog.2014.41008

[14] Ande, A.B., Omu, O.P., Ande, O.O. and Olagbuji, N.B. (2011) Features and Perceptions of Menopausal Women in Benin City. Annals of African Medicine, 10, 300-304.

[15] Eshetu, N. (2015) Knowledge, Attitudes and Associated Factors of Women Aged 30 - 49 Years towards Menopause in Gulele Sub-City of Addis Ababa, Ethiopia. (Unpublished)

[16] Ikeme, A.C.C., Okeke, T.C., Akogu, S.P.O. and Chinwuba, N. (2011) Knowledge and Perception of Menopause and Climacteric Symptoms among a Population of Women in Enugu, South East, Nigeria. Annals of Medicine and Health Sciences Research, 1, 31-36.

[17] Portland, M.E. and Jupiter, F.L. (2017) Pre-Menopause or Peri-Menopause. Crystal Clear Digital Marketing. Age Management Center.

[18] Stöppler, M.C. and Blocker, W. (1996-2017) Perimenopause Symptoms, Signs, Remedies, and Treatments.

[19] Pagano, T. (2016) Your Health in Postmenopause. WebMD Medical Reference. North American Menopause Society.

[20] Oermann, M.H. and Hays, J.C. (2016) Writing for Publication in Nursing. 3rd Edition, Springer Publishing Company, New York.

[21] Schwarzer, G. (2007) Meta: An R Package for Meta-Analysis. R News, 7, 40-45.

[22] Viechtbauer, W. (2010) Conducting Meta-Analyses in R with the Meta for Package. Journal of Statistical Software, 36, 1-48. https://doi.org/10.18637/jss.v036.i03

[23] R Core Team (2013) R: A Language and Environment for Statistical Computing. R Foundation for Statistical Computing, Vienna. http://www.R-project.org/

[24] Im, E.-O., Lee, B.I., Chee, W., Dormire, S. and Brown, A. (2010) Nursing Research, 59, 26-33. https://doi.org/10.1097/NNR.0b013e3181c3bd69

[25] Ramakuela, N.J. (2015) Experiences of Women, Menopause and Aging in the Rural Villages of Limpopo Province, South Africa. Women's Health \& Gynecology, 1, 1-5.

[26] Ibraheem, O.M., Oyewole, O.E. and Olaseha, I.O. (2015) Experiences and Perceptions of Menopause among Women in Ibadan South East Local Government Area, Nigeria. African Journal of Biomedical Research, 18, 81-94.

[27] Ramakuela, N.J., Tshitangano, T., Maluleke, M. and Tugli, A.K. (2015) Menopause, Culture and Sex among Rural Women. Journal of Human Ecology, 51, 220-225.

[28] Simon, J.A. and Reape, K.Z. (2009) Understanding the Menopausal Experiences of Professional Women. Menopause, 16, 73-76.

[29] Chism, L.A. (2014) Guiding Your Patients through Menopause. American Nurse 
Today.

[30] Smith, A.J., Hall, D.R. and Grove, D. (2009) Current Patient Perceptions on the Menopause: A South African Perspective. Climacteric, 8, 327-332. https://doi.org/10.1080/13697130500196817

[31] Hofnie-Hoëbes, K. and Matengu, K. (2014) Knowledge and Understanding of Menopause and Menopausal Symptoms: A Namibian Perspective. International Journal of Health Sciences, 2, 163-183.

[32] Anolue, F.C., Dike, E., Adogu, P. and Ebirim, C. (2011) Women's Experience of Menopause in Rural Communities in Orlu, Eastern Nigeria. International Journal of Gynecology and Obstetrics, 118, 31-33. https://doi.org/10.1016/j.ijgo.2012.02.014 


\section{Annexures}

\section{Appendix 1: Search Strategies}

PubMed 7 April 2017259 hits

Search strategy:

Menopaus*: as a text word (ie word in title or abstract)

OR Menopause: as a MeSH term (including all more narrow terms).

AND

(Information OR experience* OR perception ${ }^{\star}$ OR health seeking behave* OR knowledge): as a text word

OR Information Seeking Behaviour: as a MeSH term (there are no more narrow terms).

AND

(Africa* OR Algeria OR Angola OR Benin OR Botswana OR "Burkina Faso" OR Burundi OR Cameroon OR "Canary Islands" OR "Cape Verde" OR "Central African Republic" OR Chad OR Comoros OR Congo OR "Democratic Republic of Congo" OR Djibouti OR Egypt OR "Equatorial Guinea” OR Eritrea OR Ethiopia OR Gabon OR Gambia OR Ghana OR Guinea OR "Guinea Bissau” OR "Ivory Coast" OR "Cote d'Ivoire" OR Jamahiriya OR Jamahiryia OR Kenya OR Lesotho OR Liberia OR Libya OR Libia OR Madagascar OR Malawi OR Mali OR Mauritania OR Mauritius OR Mayote OR Morocco OR Mozambique OR Mocambique OR Namibia OR Niger OR Nigeria OR Principe OR Reunion OR Rwanda OR "Sao Tome" OR Senegal OR Seychelles OR "Sierra Leone" OR Somalia OR "South Africa" OR "St Helena" OR Sudan OR Swaziland OR Tanzania OR Togo OR Tunisia OR Uganda OR "Western Sahara" OR Zaire OR Zambia OR Zimbabwe OR "Central Africa" OR "Central African" OR "West Africa” OR "West African" OR "Western Africa" OR "Western African" OR "East Africa" OR "East African" OR "Eastern Africa" OR "Eastern African" OR "North Africa" OR "North African" OR "Northern Africa" OR "Northern African" OR "South African" OR "Southern Africa" OR "Southern African" OR "sub Saharan Africa" OR "sub Saharan African" OR "subSaharan Africa" OR "subSaharan African"): as text words

OR Africa: as a MeSH term (there are no more narrow terms)

BioMed Central 19 April 201785 hits.

Menopause AND information seeking AND (South Africa* OR Namibia*)

As text words

Sorted by "relevance" and browsed for studies since 2000. No relevant studies.

Continent specific databases: 7 April 2017.Search strategy: Search for menopaus ${ }^{*}$ in the title. One additional hit, not identified in other databases (Saka 2011)

https://www.africabib.org/ 
African Index Medicus; http://indexmedicus.afro.who.int/

African Journals Online (AJOL) https://www.ajol.info/

Embase 19 April 2017250 hits.

Database(s): EMBASE 1947-Present [OVID]

Search Strategy:

Key to terms used:

I = indexer-provided subject heading

$\mathrm{kw}=$ author keyword

$\mathrm{tw}=$ word in the title or abstract

\begin{tabular}{|c|c|c|}
\hline \# & Searches & Results \\
\hline 1 & exp menopause/ & 52840 \\
\hline 2 & menopaus $^{\star} . \mathrm{kw}$. & 12359 \\
\hline 3 & 1 or 2 & 56656 \\
\hline 4 & information seeking/ & 2204 \\
\hline 5 & $\begin{array}{l}\text { (Information or experience }{ }^{*} \text { or perception }{ }^{*} \text { or health seeking behave }{ }^{*} \text { or } \\
\text { knowledge).tw. }\end{array}$ & 3048848 \\
\hline 6 & 4 or 5 & 3049217 \\
\hline 7 & exp Africa/ & 305611 \\
\hline
\end{tabular}

7 exp Africa/

305611

(Africa* or Algeria or Angola or Benin or Botswana or Burkina Faso or Burundi or Cameroon or Canary Islands or Cape Verde or Central African Republic or Chad or Comoros or Congo or Djibouti or Egypt or Equatorial Guinea or Eritrea or Ethiopia or Gabon or Gambia or Ghana or Guinea or Guinea Bissau or Ivory Coast or Jamahiriya or Kenya or Lesotho or Liberia or Libya or Libia or Madagascar or Malawi or Mali or Mauritania or Mauritius or Mayote or Morocco or Mozambique or Mocambique or Namibia or Niger or Nigeria or Principe or

8 Reunion or Rwanda or Sao Tome or Senegal or Seychelles or Sierra Leone or Somalia or South Africa or St Helena or Sudan or Swaziland or Tanzania or Togo or Tunisia or Uganda or Western Sahara or Zaire or Zambia or Zimbabwe or Central Africa or Central African or West Africa or West African or Western Africa or Western African or East Africa or East African or Eastern Africa or Eastern African or North Africa or North African or Northern Africa or Northern African or South African or Southern Africa or Southern African or Saharan Africa or Saharan African).tw.

97 or 8

$10 \quad 3$ and 6 and 9

11 limit 10 to $\mathrm{yr}=$ “2000-2017” 250

Google Scholar 19 April 2017 Terms in search box

Menopaus* information seeking Africa*

OR

Menopaus* Namibia*

Limited to 2000 to 2017 . Then browsed through each set of results until clearly irrelevant 15 pages. 3 potentially relevant studies identified.

Medline and Medline in Process 19 April 2017143 hits 
Database(s): Ovid MEDLINE(R) without Revisions 1996 to April Week 1 2017, Ovid MEDLINE(R) In-Process \& Other Non-Indexed Citations April 18, 2017 Search Strategy:

Key to terms used:

I =indexer-provided subject heading

$\mathrm{kw}=$ author keyword

$\mathrm{tw}=$ word in the title or abstract

\begin{tabular}{|c|c|c|}
\hline$\#$ & Searches & Results \\
\hline 1 & exp menopause/ & 35116 \\
\hline 2 & menopaus*.kw. & 1607 \\
\hline 3 & 1 or 2 & 36017 \\
\hline 4 & information seeking behavior/ & 1335 \\
\hline 5 & $\begin{array}{l}\text { (Information or experience }{ }^{\star} \text { or perception }{ }^{\star} \text { or health seeking behave* or knowl- } \\
\text { edge).tw. }\end{array}$ & 1804883 \\
\hline 6 & 4 or 5 & 1805096 \\
\hline 7 & exp Africa/ & 142816 \\
\hline & $\begin{array}{l}\text { (Africa* or Algeria or Angola or Benin or Botswana or Burkina Faso or Burundi } \\
\text { or Cameroon or Canary Islands or Cape Verde or Central African Republic or } \\
\text { Chad or Comoros or Congo or Djibouti or Egypt or Equatorial Guinea or Eritrea } \\
\text { or Ethiopia or Gabon or Gambia or Ghana or Guinea or Guinea Bissau or Ivory } \\
\text { Coast or Jamahiriya or Kenya or Lesotho or Liberia or Libya or Libia or } \\
\text { Madagascar or Malawi or Mali or Mauritania or Mauritius or Mayote or Morocco } \\
\text { or Mozambique or Mocambique or Namibia or Niger or Nigeria or Principe or }\end{array}$ & \\
\hline 8 & $\begin{array}{l}\text { Reunion or Rwanda or Sao Tome or Senegal or Seychelles or Sierra Leone or } \\
\text { Somalia or South Africa or St Helena or Sudan or Swaziland or Tanzania or Togo } \\
\text { or Tunisia or Uganda or Western Sahara or Zaire or Zambia or Zimbabwe or } \\
\text { Central Africa or Central African or West Africa or West African or Western } \\
\text { Africa or Western African or East Africa or East African or Eastern Africa or } \\
\text { Eastern African or North Africa or North African or Northern Africa or Northern } \\
\text { African or South African or Southern Africa or Southern African or Saharan } \\
\text { Africa or Saharan African).tw. }\end{array}$ & 269200 \\
\hline 9 & 7 or 8 & 302023 \\
\hline 10 & 3 and 6 and 9 & 156 \\
\hline 11 & limit 10 to $y r=" 2000-2017 ”$ & 143 \\
\hline
\end{tabular}

Scopus 19 April 201754 hits.

(TITLE-ABS-KEY (menopaus*)) AND (TITLE-ABS-KEY (information OR ex-

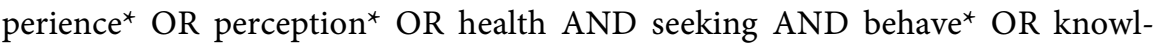
edge)) 


\section{Appendix 2: PRISMA 2009 CHECKLIST}

\begin{tabular}{lll}
\hline Section/topic $\quad \# \quad$ Checklist item & $\begin{array}{l}\text { Reported } \\
\text { on page \# }\end{array}$
\end{tabular}

Title

\section{ABSTRACT}

Structured summary

Provide a structured summary including, as applicable: background; objectives; data sources; study eligibility criteria, participants, and interventions; study appraisal and synthesis methods; results; limitations; conclusions and implications of key findings; systematic review registration number.

\section{INTRODUCTION}

Rationale

Objectives

METHODS

Protocol and

registration

Eligibility criteria

Information sources

Search

Study selection

Data collection

process

Data items

Risk of bias in

individual studies

Summary measures

Synthesis of results
1 Identify the report as a systematic review, meta-analysis, or both.
3 Describe the rationale for the review in the context of what is already known. interventions, comparisons, outcomes, and study design (PICOS).
Provide an explicit statement of questions being addressed with reference to participants,

Indicate if a review protocol exists, if and where it can be accessed (e.g., Web address), and, if available, provide registration information including registration number.

Specify study characteristics (e.g., PICOS, length of follow-up) and report characteristics (e.g., years considered, language, publication status) used as criteria for eligibility, giving rationale.

Describe all information sources (e.g., databases with dates of coverage, contact with study authors to identify additional studies) in the search and date last searched.

Present full electronic search strategy for at least one database, including any limits used, such that it could be repeated.

State the process for selecting studies (i.e., screening, eligibility, included in systematic review, and, if applicable, included in the meta-analysis).

Describe method of data extraction from reports (e.g., piloted forms, independently, in duplicate) and any processes for obtaining and confirming data from investigators.

List and define all variables for which data were sought (e.g., PICOS, funding sources) and any assumptions and simplifications made.

Describe methods used for assessing risk of bias of individual studies (including specification of

12 whether this was done at the study or outcome level), and how this information is to be used in any data synthesis.

13 State the principal summary measures (e.g., risk ratio, difference in means).

Describe the methods of handling data and combining results of studies, if done, including measures
1 and 3

2 and 15;

Table 1

Table 2 
Books, magazines, journals

\begin{tabular}{|c|c|c|c|c|c|c|c|c|}
\hline Study & Events & Total & & & ortion & $95 \%-\mathrm{Cl}$ & $\begin{array}{r}\text { Weight } \\
\text { (fixed) }\end{array}$ & $\begin{array}{r}\text { Weight } \\
\text { (random) }\end{array}$ \\
\hline Tsehay 2014 & 176 & 263 & & $\rightleftarrows$ & 0.67 & {$[0.61 ; 0.73]$} & $13.3 \%$ & $12.7 \%$ \\
\hline Bello 2016 & 43 & $352 \div$ & & & 0.12 & {$[0.09 ; 0.16]$} & $8.7 \%$ & $12.6 \%$ \\
\hline Smith 2009 & 150 & 392 & $\div$ & & 0.38 & {$[0.33 ; 0.43]$} & $21.2 \%$ & $12.8 \%$ \\
\hline Ibraheem 2015 & 21 & $240 \div$ & & & 0.09 & {$[0.05 ; 0.13]$} & $4.4 \%$ & $12.4 \%$ \\
\hline Hofnie 2009 & 140 & 1076 & & & 0.13 & {$[0.11 ; 0.15]$} & $27.9 \%$ & $12.8 \%$ \\
\hline Osinowo 2003 & 8 & 45 & & & 0.18 & {$[0.08 ; 0.32]$} & $1.5 \%$ & $11.5 \%$ \\
\hline Eshetu 2015 & 87 & 586 & & & 0.15 & {$[0.12 ; 0.18]$} & $17.0 \%$ & $12.8 \%$ \\
\hline Saka 2011 & 28 & $400 \div$ & & & 0.07 & {$[0.05 ; 0.10]$} & $6.0 \%$ & $12.5 \%$ \\
\hline Fixed effect model & & 3354 & & & 0.22 & {$[0.20 ; 0.23]$} & $100.0 \%$ & -- \\
\hline Random effects model & & $\leq$ & & & 0.19 & {$[0.10 ; 0.33]$} & -- & $100.0 \%$ \\
\hline
\end{tabular}

$\begin{array}{lllllll}0.1 & 0.2 & 0.3 & 0.4 & 0.5 & 0.6 & 0.7\end{array}$

Friends

Study

Tsehay 2014

Bello 2016

Osinowo 2003

Eshetu 2015

Saka 2011

Anolue 2012

Maharaj 2007

Fixes effect model

Random effects model

Heterogeneity: $f^{2}=94 \%, \mathrm{t}^{2}=0$

\section{Events Total}

$187 \quad 263$

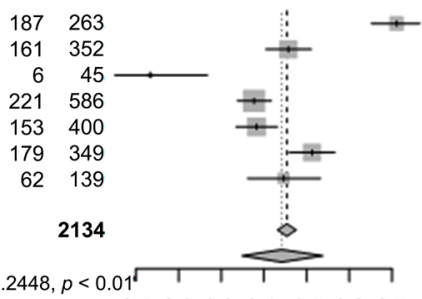

Weight Weight

Proportion $\quad 95 \%-\mathrm{Cl}$ (fixed) (random)

$0.71[0.65 ; 0.77] \quad 10.8 \% \quad 14.9 \%$

$0.46[0.40 ; 0.51] \quad 17.5 \% \quad 15.3 \%$

$0.13[0.05 ; 0.27] \quad 1.0 \% \quad 9.0 \%$

$0.38[0.34 ; 0.42] \quad 27.5 \% \quad 15.6 \%$

$0.38[0.33 ; 0.43] \quad 18.9 \% \quad 15.4 \%$

$0.51[0.46 ; 0.57] \quad 17.4 \% \quad 15.3 \%$

$0.45[0.36 ; 0.53] \quad 6.9 \% \quad 14.4 \%$

$\begin{array}{lrr}0.45[0.43 ; 0.48] & 100.0 \% & - \\ 0.44[0.35 ; 0.54] & -- & 100.0 \%\end{array}$

\section{Medical providers}

Study

Tsehay 2014

Bello 2016

Ikeme 2011

Smith 2009

Dienye 2013

Ibraheem 2015

Hodnie 2009

Eshetu 2015

Saka 2011

Fixes effect model

Random effects mode

Heterogeneity: $l^{2}=99 \%, t^{2}=1.665, p<0.01$
Events Total

$$
181263
$$

$\begin{array}{rr}181 & 263 \\ 60 & 352 \\ 192 & 432\end{array}$

$\begin{array}{ll}192 & 432 \\ 342 & 392\end{array}$

$\begin{array}{rr}342 & 392 \\ 172 & 385\end{array}$

12240

1291076

122586

$38 \quad 400$

4126

6

1

$\begin{array}{lllllll}1 & 0.2 & 0.3 & 0.4 & 0.5 & 0.6 & 0.7\end{array}$ 
Religious sources

Study

Ikeme 2011 Dienye 2013 Ibraheem 2015 Hofnie 2009

Anolue 2012

Fixes effect model Random effects model

Heterogeneity: $I^{2}=97 \%, t^{2}=1.246, p<0.01$

Events Total

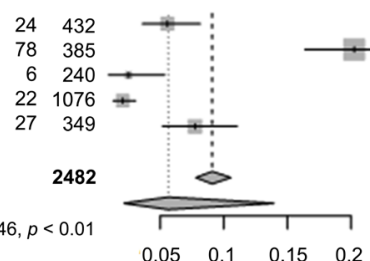

Tv and Radio
Events Total

$$
\begin{array}{rr}
165 & 263 \\
43 & 352 \\
32 & 1076 \\
86 & 586 \\
58 & 139 \\
& \\
& 2416
\end{array}
$$$$
321076=
$$

Hofnie 2009

Eshetu 2015

Maharaj 2007

Fixes effect model

Random effects mode

Heterogeneity: $f^{2}=99 \%, \mathrm{t}^{2}=2.233, p<0.01$ $\begin{array}{llllll}0.1 & 0.2 & 0.3 & 0.4 & 0.5 & 0.6\end{array}$
Proportion $\quad 95 \%-\mathrm{Cl} \quad \begin{aligned} & \text { Weight } \\ & \text { (fixed) }\end{aligned}$ (random)

$0.06[0.04 ; 0.08] \quad 16.5 \% \quad 20.3 \%$

$0.20[0.16 ; 0.25] \quad 45.3 \% \quad 20.7 \%$

$0.02[0.01 ; 0.05] \quad 4.3 \% \quad 18.5 \%$ $0.02[0.01 ; 0.03] \quad 15.7 \% \quad 20.2 \%$

$0.08[0.05 ; 0.11] \quad 18.2 \% \quad 20.3 \%$

$0.09[0.08 ; 0.11] 100.0 \%$ $0.06[0.02 ; 0.14]$ Proportion $\quad 95 \%-\mathrm{Cl} \quad \begin{aligned} & \text { Weight } \\ & \text { (fixed) }\end{aligned} \begin{gathered}\text { Weight } \\ \text { (random) }\end{gathered}$ 